\title{
ON THE CUBIC NLS ON 3D COMPACT DOMAINS
}

\author{
FABRICE PLANCHON
}

\begin{abstract}
We prove bilinear estimates for the Schrödinger equation on 3D domains, with Dirichlet boundary conditions. On non-trapping domains, they match the $\mathbb{R}^{3}$ case, while on bounded domains they match the generic boundaryless manifold case. As an application, we obtain global wellposedness for the defocusing cubic NLS for data in $H_{0}^{s}(\Omega), 1<s \leqslant 3$, with $\Omega$ any bounded domain with smooth boundary.
\end{abstract}

\section{Résumé}

On démontre des estimations bilinéaires pour l'équation de Schrödinger sur des domaines tridimensionnels, avec condition de Dirichlet au bord. Dans le cas non-captant, on retrouve les estimations connues dans $\mathbb{R}^{3}$, et sur un domaine borné on obtient des estimations similaires à celles du cas d'une variété compacte générique sans bord. Une application est donnée à l'existence de solutions globales dans $H_{0}^{s}(\Omega), 1<s \leqslant 3$, avec $\Omega$ un domaine borné régulier.

\section{INTRODUCTION}

Let $\Omega \subset \mathbb{R}^{3}$ be a domain with a smooth boundary $\partial \Omega$, and consider the Schrödinger equation

$$
i \partial_{t} \phi+\Delta \phi=\varepsilon|\phi|^{2} \phi, \text { with } \phi_{\mid \partial \Omega}=0 \text { and } \phi_{t=0}=\phi_{0} .
$$

Our first interest is the linear equation, that is $\varepsilon=0$. When $\Omega=\mathbb{R}^{3}$, dispersive properties of (1) are well-understood and play a crucial role in understanding the nonlinear case $\varepsilon= \pm 1$. One has a large set of sharp Strichartz estimates $([32,16,23$, and among other things the nonlinear problem is locally well-posed in the Sobolev space $\dot{H}^{\frac{1}{2}}\left(\mathbb{R}^{3}\right)([13])$, and globally well-posed in the energy space $H^{1}\left(\mathbb{R}^{3}\right)$ (with a smallness condition on the $L^{2}\left(\mathbb{R}^{3}\right)$ norm in the focusing case $\varepsilon=-1$ ), see [16]. On domains, our understanding of dispersion is far from complete: depending on the geometry of light rays, we have essentially two cases,

- $\Omega$ is the exterior of a non trapping obstacle: a restricted set of non sharp (but scale-invariant) Strichartz estimates is known to hold ([3], see also [28] and [9]), together with square-function type estimates, 22. In the special case of a strictly convex obstacle, Strichartz estimates hold as in $\mathbb{R}^{3}$ (except possibly for the endpoint), [20];

- $\Omega$ is a compact domain with smooth boundaries: there, the same set of estimates as in the exterior case in known to hold, but only at semi-classical time scales. In fact, the estimates in the non trapping case are obtained by combining these semi-classical estimates with the local smoothing effect, following a strategy pioneered by [31. Thus, the best available Strichartz estimates are due to [3] (see also [4 and [2] for earlier progress) and they exhibit losses with respect to scaling (translating to a regularity loss when compared to the exterior case). Moreover, when compared to Strichartz estimates on compact manifolds without boundaries ([10]), we have a restricted set of exponents. As such, one may solve the nonlinear cubic equation (1) only for $H^{s}(\Omega) \cap H_{0}^{1}(\Omega), 1<s \leqslant 2$, and this is a local in time construction (combined logarithmic losses in the available Strichartz estimates prevent a Brezis-Gallouët or Yudovitch-like argument). Moreover, counterexamples to the sharp Strichartz estimates

Date: August 16, 2018.

The author was partially supported by A.N.R. grant SWAP. 
were constructed in [19] (though this leaves a large gap to be filled between our current knowledge and these counterexamples).

On compact, boundary less, manifolds, an alternate strategy was pushed forward in [11, 12]: Strichartz estimates are replaced by bilinear $L_{t, x}^{2}$ estimates, following [6] which dealt with rational tori. On specific manifolds (e.g. Zoll manifolds), for which eigenvalues and eigenfunctions are well-distributed, these bilinear estimates prove to be more efficient than Strichartz estimates. In [1], such bilinear estimates are established for radial data on $\Omega=B(0,1)$, and they match the Zoll manifold case (however, [1] provides a counter-example to such optimal estimates in the non radial case), providing near optimal well-posedness for the cubic NLS. Moreover, we learned after completion of the present work that the radial quintic NLS may be addressed ([26]) by tranfering estimates from the boundary less manifold $\mathbb{S}^{3}$. In a different direction, bilinear estimates have proved to be quite useful in the whole space when dealing with long time behavior of nonlinear solutions (from [7] to [14]). Heuristically, one would expect such bilinear estimates to hold on the semiclassical time scale for generic compact manifolds, and this is indeed true, [18].

Our aim is to obtain such estimates on generic domains, with Dirichlet boundary conditions. In [28], we derived a linear $L_{t, x}^{4}$ estimate from our general bilinear virial machinery (later matched in [3], among a larger set of estimates); as a side note, [28] provides an argument allowing to recover [7] from the Radon transform estimate, but such an argument, while mostly based on integration by parts, is still using preservation of the Fourier support by the linear flow (and the argument is, in some sense, non local due to the use of the Radon transform). In [27], we provide a different argument, much more local, and based on a Sobolev trace lemma (such an argument would allow to recover [18, through an appropriate microlocalization procedure); similar arguments appeared independently in [29] (see also [30] for further developments) in dealing with the covariant Schrödinger equation. However these procedures still rely on localizing the Fourier support in a specific direction, which seems out of reach for the boundary value case. Very recently, however, 15] obtained a variant of such bilinear estimates on the exterior of the strictly convex obstacle (such a gradient form of bilinear estimates already proved useful in [1]). The argument from [15] relies on the Strichartz estimates from [20] and on the trace lemma applied to the bilinear virial estimates from [28], but done in a way which only involve the low frequencies, bypassing "directional" requirements on the high frequencies. In the present paper, we adapt that argument to generic domains, irrespective of the geometry of the boundary, obtaining a bilinear estimate which involves trace terms on the right-hand side. Such trace terms are then disposed of, either by local smoothing effects in the non trapping case, or by restricting to semiclassical time scales on compact domains. Furthermore, we utilize the machinery developed in [21] to derive the usual bilinear estimate from the gradient one. The combination of both estimates immediately recovers the local well-posedness result we mentioned earlier for (11) in $H^{s}(\Omega) \cap H_{0}^{1}(\Omega)$ for $1<s \leqslant 2$ ([1]) ; we further develop the local theory close to $s=1$ by combining our bilinear estimate, the endpoint Strichartz estimate from [3] together with a discrete Gronwall-like estimate (inspired by [5]) to obtain global well-posedness results.

The main advantage of the present note is that it mostly relies on basic tools to obtain estimates on linear solutions, as we do not require any sophisticated microlocal approximation of the solution; however, we deal with the flat Laplacian on a domain, unlike most of the aforementioned results which deal with smooth manifolds (hence variable coefficients) and both Dirichlet and Neumann boundary conditions. We claim however that the present approach is mostly local in space, and may be adapted to the variable coefficients case, at the expense of technical tedious complications. We chose to focus on the flat case to highlight the simplicity of the argument. 


\section{MAin RESUlts}

2.1. Bilinear estimates for the linear Schrödinger equation on a domain $\Omega$. Let $\Omega \subset \mathbb{R}^{3}$ be a domain with a smooth boundary $\partial \Omega$, and consider the Schrödinger equation

$$
i \partial_{t} w_{m}+\Delta w_{m}=0, \text { with } w_{m \mid \partial \Omega}=0,
$$

where $m$ stands for data $w_{m}(0)$ which are spectrally localized at $\sqrt{-\Delta} \sim 2^{m}, m \in \mathbb{Z}$. By this, we mean that, for some $\varphi \in C_{0}^{\infty}\left(\mathbb{R}_{+}\right), w_{m}=\varphi\left(2^{-m} \sqrt{-\Delta}\right) w_{m}$. The smoothed out spectral projector $\varphi(\sqrt{-\Delta})$ may be defined by spectral calculus or, more directly, by the Dynkin-Helffer-Sjöstrand formula (see [21] for details and useful properties of such operators). Let us define, for $k \in \mathbb{N}^{\star}$ and with $\partial_{n}$ the normal derivative on the boundary,

$$
\mathcal{H}_{k}\left(w_{m}\right)=\left\|w_{m}(0)\right\|_{2}\left\|w_{m}(0)\right\|_{\dot{H}_{0}^{1}(\Omega)}+\int_{0}^{T} \int_{x \in \partial \Omega} \sum_{l=0}^{k} 2^{-2 l m}\left|\partial_{n} \Delta^{l} w_{m}\right|^{2} d S_{x} d t .
$$

Theorem 2.1. Consider two solutions $u_{j}$ and $v_{k}$ to (1), with data $u_{j}(0)$ which is spectrally localized at $\sqrt{-\Delta} \sim 2^{j}$ and data $v_{k}(0)$ which is spectrally localized at $\sqrt{-\Delta} \sim 2^{k}$, where $k \leqslant j$. Then the following bilinear estimate holds:

$$
\int_{0}^{T}\left\|v_{k} \nabla u_{j}\right\|_{2}^{2} d t \lesssim 2^{2 k}\left(\left\|v_{k}(0)\right\|_{2}^{2} \mathcal{H}_{0}\left(u_{j}\right)+\left\|u_{j}(0)\right\|_{2}^{2} \mathcal{H}_{2}\left(v_{k}\right)\right)
$$

We now provide bilinear estimates when one has global (in time) control of the boundary term.

Proposition 2.1. Let $u_{j}$ and $v_{k}$ be as in Theorem 2.1 and assume moreover that $\Omega=\mathbb{R}^{3} \backslash \Sigma$, where $\Sigma$ is a non trapping smooth compact obstacle. Then,

$$
\int_{\mathbb{R}}\left\|u_{j} v_{k}\right\|_{2}^{2} d t+2^{-2 j} \int_{\mathbb{R}}\left\|v_{k} \nabla u_{j}\right\|_{2}^{2} d t \lesssim 2^{2 k-j}\left\|u_{j}(0)\right\|_{2}^{2}\left\|v_{k}(0)\right\|_{2}^{2} .
$$

In [15], the gradient estimate from the left-hand side of (5) is obtained for a strictly convex $\Sigma$ (actually the statement there deals with $\left\|\nabla\left(\bar{v}_{k} u_{j}\right)\right\|_{2}$ but as we will see later the difference term is at a lower order).

On a bounded domain, one will have losses unless $T \lesssim 2^{-j}$ :

Proposition 2.2. Let $u_{j}$ and $v_{k}$ be as in Theorem [2.1 and assume moreover that $\Omega$ is a compact domain of $\mathbb{R}^{3}$ with smooth boundary. If $T<2^{-j}$ then

$$
\int_{0}^{T}\left\|u_{j} v_{k}\right\|_{2}^{2} d t+2^{-2 j} \int_{0}^{T}\left\|v_{k} \nabla u_{j}\right\|_{2}^{2} d t \lesssim 2^{2 k-j}\left\|u_{j}(0)\right\|_{2}^{2}\left\|v_{k}(0)\right\|_{2}^{2} .
$$

Moreover, for $T<+\infty$, we have

$$
\int_{0}^{T}\left\|u_{j} v_{k}\right\|_{2}^{2} d t+2^{-2 j} \int_{0}^{T}\left\|v_{k} \nabla u_{j}\right\|_{2}^{2} d t \lesssim T 2^{2 k}\left\|u_{j}(0)\right\|_{2}^{2}\left\|v_{k}(0)\right\|_{2}^{2} .
$$

\section{Application}

We now return to our nonlinear cubic Schrödinger equation (11).

Theorem 3.1. Let $\Omega$ be a bounded domain in $\mathbb{R}^{3}$ with smooth boundary. Let $\phi_{0} \in H_{0}^{s}(\Omega)$ with $1<s \leqslant 3$. Then the defocusing (1) admits a global solution $u \in C_{t}\left(H_{0}^{s}\right)$ which is unique in a suitable subspace. The same result holds true in the focusing case provided the $L^{2}(\Omega)$ norm of $\phi_{0}$ is sufficiently small with respect to the $H_{0}^{1}(\Omega)$ norm. Moreover, the associated flow is analytic. 
Before stating further results, we need to define several function spaces which will be of help. We start with (inhomogeneous) Besov spaces which are built on the spectral localization (21] for details). Let us recall that a Littlewood-Paley decomposition is a collection of operator $\left(\Delta_{j}\right)_{j \in \mathbb{N}}$ defined as follows: let $\phi \in \mathcal{S}(\mathbb{R})$ such that $\hat{\phi}=1$ for $|\xi| \leqslant 1$ and $\widehat{\phi}=0$ for $|\xi|>11 / 10, \phi_{j}(\xi)=$ $2^{j} \phi\left(2^{j} \xi\right), S_{j}=\phi_{j}(\sqrt{-\Delta}), \psi_{j}(\xi)=\left(\phi_{j+1}-\phi_{j}\right)(\xi), \Delta_{j}=S_{j+1}-S_{j}=\psi_{j}(\sqrt{-\Delta})$. For notational convenience, we may sometimes refer to $S_{0}$ as $\Delta_{-1}$. We shall denote $u_{j}=\Delta_{j} u$.

Definition 3.1. Let $f$ be in $\mathcal{S}^{\prime}(\Omega), s \in \mathbb{R}$ and $1 \leqslant p, q \leqslant+\infty$. We say $f$ belongs to $B_{p}^{s, q}$ (resp. $B_{p, l}^{s, q}$ ) if and only if, with $\lambda_{j, s}=2^{j s}\left(\right.$ resp. $\left.\lambda_{j, s}=2^{j s} \log ^{\frac{1}{2}} j\right)$

- $S_{0} f \in L^{p}$.

- The sequence $\left(\varepsilon_{j}\right)_{j \in \mathbb{N}}$ with $\varepsilon_{j}=\lambda(j, s)\left\|\Delta_{j}(f)\right\|_{L^{p}}$ belongs to $l^{q}$.

We define our Sobolev spaces $H_{0}^{s}(\Omega)=B_{2}^{s, 2}$ and remark $H_{0}^{1}(\Omega)$ is the usual Sobolev space associated to the Dirichlet Laplacian. We will later use its subspace $\dot{B}_{2, l}^{1,1}$. Notice that functions in $B_{2}^{s, 2}$ for $s>1 / 2$ will satisfy trace conditions (involving only powers of the Laplacian) as they are embedded in the definition through the use of the spectral projector. Finally, we will also need the conormal spaces introduced in [24, 25]. In the present paper, the atomic spaces $U^{p}$ and $V^{p}$ may be seen as a black box for which we refer to Section 2, [17, for definitions and useful properties. In our setting, we will be using Definition 2.15 in [17] with $S(t)=\exp (i t \Delta)$ the linear Schrödinger group associated with the Dirichlet Laplacian:

$$
\begin{aligned}
& U_{S}^{p}=S(\cdot) U^{p}, \quad \text { with norm }\|u\|_{U_{S}^{p}}=\|S(-\cdot) u\|_{U^{p}} \\
& V_{S}^{p}=S(\cdot) V^{p}, \quad \text { with norm }\|u\|_{V_{S}^{p}}=\|S(-\cdot) u\|_{V^{p}}
\end{aligned}
$$

Definition 3.2. Let $u(t, x) \in \mathcal{S}^{\prime}(\mathbb{R} \times \Omega), s \in \mathbb{R}$. We say that $u \in X_{l}^{1}$ if and only if,

$$
\left.\|u\|_{X_{l}^{1}}=\sum_{j \geqslant 0} 2^{j}(\log j)^{\frac{1}{2}} \| \Delta_{j} u(t, \cdot)\right) \|_{U_{S}^{2}}<+\infty
$$

and $u \in X^{s}$ if and only if,

$$
\left.\|u\|_{X^{s}}^{2}=\sum_{j \geqslant 0} 2^{2 s j} \| \Delta_{j} u(t, \cdot)\right) \|_{U_{S}^{2}}^{2}<+\infty .
$$

Similarly, we say that $v \in Y_{l}^{ \pm 1}$ if and only if,

$$
\left.\|v\|_{Y_{l}^{ \pm 1}}^{2}=\sup _{j \geqslant 0} 2^{ \pm j}(\log j)^{-\frac{1}{2}} \| \Delta_{j} v(t, \cdot)\right) \|_{V_{S}^{2}}<+\infty
$$

and $v \in Y^{s}$ if and only if,

$$
\left.\|v\|_{Y^{s}}=\sum_{j \geqslant 0} 2^{2 s j} \| \Delta_{j} v(t, \cdot)\right) \|_{V_{S}^{2}}^{2}<+\infty
$$

The most important property relating our spaces is that the Duhamel operator associated to $S(t)$ maps the dual of $Y_{l}^{-1}$ (or $Y^{-s}$ ) to $X_{l}^{1}$ (or $X^{s}$ ). Moreover, any multilinear estimate on products of linear solutions to the Schrödinger equation may be transferred to our functional spaces, at most at a cost of a log loss in the constants.

Now, we can reformulate a more precise local (in time) version of Theorem 3.1

Proposition 3.1. Let $\Omega$ be a bounded domain in $\mathbb{R}^{3}$ with smooth boundary. Let $\phi_{0} \in B_{2, l}^{1,1} \subset H_{0}^{1}(\Omega)$. Then (11) admits a unique local in time solution $u \in X_{l}^{1}$. Moreover, the associated flow is analytic, and the local time of existence $T$ is at least comparable to $\left\|\phi_{0}\right\|_{B_{2, l}^{1,1}}^{-2}$.

When the data $\phi_{0}$ is smoother, we may refine our local result: 
Proposition 3.2. Let $\Omega$ be a bounded domain in $\mathbb{R}^{3}$ with smooth boundary. Let $\phi_{0} \in H_{0}^{s}(\Omega)$ for $1<s \leqslant 3$. Then (11) admits a unique local in time solution $u \in X_{l}^{1}$. Moreover, the solution is in $X^{s} \hookrightarrow C_{T}\left(H^{s}(\Omega)\right)$, and the local time of existence $T$ is of order $\left(\log \left\|\phi_{0}\right\|_{H^{s}(\Omega)} \log \log \left\|\phi_{0}\right\|_{H^{s}(\Omega)}\right)^{-1}$ (provided the $L^{2}(\Omega)$ norm of $\phi_{0}$ is small in the focusing case).

This last result takes into account the conservation of the Hamiltonian, which provides control of the $H_{0}^{1}$ norm. The double logarithm appearing in the time of existence translates into a triple exponential growth of the corresponding Sobolev norm as a function of time.

\section{Proofs AND FURTher DEVELOPMENTS}

We start with proving Theorem 2.1. Let us recall a result from [28]. Let $u, v$ be two solutions to the Schrödinger equation which are not necessarily spectrally localized, and define

$$
I_{\rho}(u, v)=\int \rho(x-y)|u|^{2}(x)|v|^{2}(y) d x d y .
$$

Theorem 4.1 ([28]). Let $\rho$ be a weight function such that its Hessian $H_{\rho}$ is positive; let

$$
F(u, v)(x, y)=\bar{v}(y) \nabla_{x} u(x)+u(x) \nabla_{y} \bar{v}(y) .
$$

We have

$$
\begin{aligned}
\partial_{t}^{2} I_{\rho}=4 \int H_{\rho}(x-y) & (F(u, v)(x, y), \bar{F}(u, v)(x, y)) d x d y \\
-2 \int_{x \in \partial \Omega, y \in \Omega}|v|^{2}(y) \partial_{n} \rho(x-y)\left|\partial_{n} u\right|^{2}(x) d S_{x} d y & -2 \int_{y \in \partial \Omega, x \in \Omega}|u|^{2}(x) \partial_{n} \rho(x-y)\left|\partial_{n} v\right|^{2}(y) d S_{y} d x
\end{aligned}
$$

We follow [15] in spirit, although our presentation will differ, both on the choice of weight and our later treatment of lower order terms. First, rather than applying our theorem with the weight $\left.\rho_{\omega}(x-y)=\mid \omega \cdot(x-y)+\sigma\right) \mid$ where $\omega \in \mathbb{S}^{2}$ is any direction and $\sigma \in \mathbb{R}$ is a spatial parameter which will be averaged later, we directly use the weight $\rho_{\omega, k}$ defined as follows:

$$
\begin{aligned}
& \rho_{\omega, k}(z)=|\omega \cdot z| \text { if }|\omega \cdot z|>2^{-k}, \\
& \rho_{\omega, k}(z)=2^{k}|\omega \cdot z|^{2} / 2+2^{-k} / 2 \text { if }|\omega \cdot z| \leqslant 2^{-k},
\end{aligned}
$$

This yields,

$$
\begin{aligned}
& \int_{|(x-y) \cdot \omega|<2^{-k}}\left|u_{j}(x)(\omega \cdot \nabla) \bar{v}_{k}(y)+\bar{v}_{k}(y)(\omega \cdot \nabla) u_{j}(x)\right|^{2} d x d y \\
&-\int_{x \in \partial \Omega, y \in \Omega}\left|v_{k}\right|^{2}(y) \partial_{n} \rho_{\omega, k}(x-y)\left|\partial_{n} u_{j}\right|^{2}(x) d S_{x} d y \\
& \quad-\int_{y \in \partial \Omega, x \in \Omega}\left|u_{j}\right|^{2}(x) \partial_{n} \rho_{\omega, k}(x-y)\left|\partial_{n} v_{k}\right|^{2}(x) d S_{y} d x=\frac{1}{4} \partial_{t}^{2} I_{\rho_{\omega}, k} .
\end{aligned}
$$

Let us deal with the right-hand side: after time integration and with

$$
\omega \cdot \nabla \rho_{\omega, k}(z)=\mathbf{1}_{|z \cdot \omega|>2^{-k}} \operatorname{sgn}(\mathrm{z} \cdot \omega)+\mathbf{1}_{|z \cdot \omega| \leqslant 2^{-k}} 2^{k}|z \cdot \omega|
$$


which we notice is a bounded function, we get

$$
\begin{aligned}
\partial_{t} I_{\rho_{\omega, k}}(t)=\int \omega \cdot \nabla \rho_{\omega, k}(x-y) \operatorname{Im} \bar{u}_{j}(\omega \cdot \nabla) & u_{j}(x)\left|v_{k}\right|^{2}(y) d x d y \\
& -\int \omega \cdot \nabla \rho_{\omega, k}(x-y) \operatorname{Im} \bar{v}_{k}(\omega \cdot \nabla) v_{k}(y)\left|u_{j}\right|^{2}(x) d x d y
\end{aligned}
$$

which is easily seen to be bounded,

$$
\left|\partial_{t} I_{\rho_{\omega, k}}(T)-\partial_{t} I_{\rho_{\omega, k}}(0)\right| \lesssim\left\|v_{k}(0)\right\|_{2}^{2}\left\|u_{j}(0)\right\|\left\|_{2}\right\| u_{j}(0)\left\|_{\dot{H}_{0}^{1}(\Omega)}+\right\| u_{j}(0)\left\|_{2}^{2}\right\| v(0)\left\|_{2}\right\| v_{k}(0) \|_{\dot{H}_{0}^{1}(\Omega)} .
$$

On the other hand, $\left|\partial_{n} \rho_{\omega, k}\right| \leqslant 1$ and the boundary terms in (15) may be bounded by

$$
\begin{aligned}
\int_{\partial \Omega \times \Omega}\left|v_{k}\right|^{2}(y)\left|\partial_{n} \rho_{\omega}(x-y)\right|\left|\partial_{n} u_{j}\right|^{2}(x) d S_{x} d y \\
\quad+\int_{\Omega \times \partial \Omega}\left|u_{j}\right|^{2}(x)\left|\partial_{n} \rho_{\omega}(x-y)\left\|\left.\partial_{n} v_{k}\right|^{2}(x) d S_{y} d x \lesssim \mathcal{H}_{0}\left(u_{j}\right)\right\| v_{k}(0)\left\|_{2}^{2}+\mathcal{H}_{0}\left(v_{k}\right)\right\| u_{j}(0) \|_{2}^{2} .\right.
\end{aligned}
$$

We are thus left with

$$
\begin{aligned}
\int_{0}^{T} \int_{|(x-y) \cdot \omega|<2^{-k}}\left|u_{j}(x)(\omega \cdot \nabla) \bar{v}_{k}(y)+\bar{v}_{k}(y)(\omega \cdot \nabla) u_{j}(x)\right|^{2} d x d y d t & \\
& \quad \lesssim\left\|v_{k}(0)\right\|_{2}^{2} \mathcal{H}_{0}\left(u_{j}\right)+\left\|u_{j}(0)\right\|_{2}^{2} \mathcal{H}_{0}\left(v_{k}\right) .
\end{aligned}
$$

We now consider the term $u_{j}(\omega \cdot \nabla) \bar{v}_{k}$ in the $|\cdots|^{2}$ inside (17) above, and restrict to $\left|(x-y)^{\perp}\right|<2^{-k}$, where for any $z, z^{\perp}=z-(z \cdot \omega) \omega$, to get

$$
\int_{0}^{T} \int_{|(x-y) \cdot \omega|<2^{-k}} \int_{\left|(x-y)^{\perp}\right|<2^{-k}}\left|u_{j}(x)(\omega \cdot \nabla) \bar{v}_{k}(y)\right|^{2} d x d y d t
$$

changing variables so that $y=x+z$, we may bound this integral from above by

$$
J_{\omega}=\int_{0}^{T} \int_{|z|<2^{-(k-1)}}\left|u_{j}(x)(\omega \cdot \nabla) v_{k}(x+z)\right|^{2} d x d z d t,
$$

as $\left\{|(x-y) \cdot \omega|<2^{-k}\right\} \cap\left\{\left|(x-y)^{\perp}\right|<2^{-k}\right\} \subset\left\{|x-y|<2^{-(k-1)}\right\}$. Notice that the variable $z$ is averaged over a ball of size $2^{-(k-1)}$, which is the dual size of the spectral localization at $2^{k}$ of $v_{k}$. This will be crucial later on. For now, by Cauchy-Schwarz,

$$
J_{\omega}^{2} \lesssim \int_{0}^{T}\left\|u_{j}\right\|_{4}^{4} d x d t \int_{0}^{T}\left\|\nabla v_{k}\right\|_{4}^{4} d x d t \int_{|z|<2^{-(k-1)}} d z \lesssim 2^{-3 k} \int_{0}^{T}\left\|u_{j}\right\|_{4}^{4} d x d t \int_{0}^{T}\left\|\sqrt{-\Delta} v_{k}\right\|_{4}^{4} d x d t .
$$

We then use the linear $L_{t, x}^{4}$ estimate from [28], which becomes with our notations

Lemma 4.1 ([28]). Let $w_{m}$ be a solution to (2) . Then

$$
\int_{0}^{T}\left\|w_{m}\right\|_{4}^{4} d t \lesssim\left\|w_{m}(0)\right\|_{2}^{2} \mathcal{H}_{0}\left(w_{m}\right)
$$

Moreover, when $\Omega$ is compact,

$$
\int_{0}^{T}\left\|w_{m}\right\|_{4}^{4} d t \lesssim T 2^{2 m}\left\|w_{m}(0)\right\|_{2}^{4}
$$

Therefore, we have $\int_{0}^{T}\left\|u_{j}\right\|_{4}^{4} d t \lesssim\left\|u_{j}(0)\right\|_{2}^{2} \mathcal{H}_{0}\left(u_{j}\right)$ and $\int_{0}^{T}\left\|v_{k}\right\|_{4}^{4} d t \lesssim\left\|v_{k}(0)\right\|_{2}^{2} \mathcal{H}_{0}\left(v_{k}\right)$, and get

$$
J_{\omega} \lesssim\left\|u_{j}(0)\right\|_{2} \mathcal{H}_{0}\left(u_{j}\right) \times 2^{-3 k+2 k}\left\|v_{k}(0)\right\|_{2} \mathcal{H}_{0}\left(v_{k}\right) .
$$


Going back to (17), we may restrict the space integration to $|x-y|<2^{-k}$ as well as change variables $y=x+z$ and consequently we are left with

$$
K_{\omega}(u, v)=\int_{0}^{T} \int_{|z|<2^{-k}}\left|v_{k}(x+z)(\omega \cdot \nabla) u_{j}(x)\right|^{2} d x d z d t
$$

for which we proved (combining (17) and (20)

$$
K_{\omega}(u, v) \lesssim 2^{-k}\left(\left\|v_{k}(0)\right\|_{2}^{2} \mathcal{H}_{0}\left(u_{j}\right) \mid+\left\|u_{j}(0)\right\|_{2} \mathcal{H}_{0}\left(v_{k}\right)\right) .
$$

We digress with an elementary version of the trace lemma:

Lemma 4.2. Let $\phi$ be a smooth function in $\mathbb{R}^{3}$ and $\lambda>0$. Denote by $C_{\mu}$ the cube centered at $x=0$ with size $\mu$. Then

$$
|\phi(0)|^{2} \lesssim \lambda^{-1} \int_{C_{\lambda^{-1}}}|\Delta \phi|^{2}+\lambda^{3} \int_{C_{\lambda^{-1}}}|\phi|^{2}
$$

By standard elliptic regularity, (22) is true for a cube of size $\lambda=1$. The estimate for any $\lambda$ then follows by rescaling, applying the estimate for $\lambda=1$ to $\phi\left(\lambda^{-1} \cdot\right)$.

We now apply the lemma to $v_{k}(x+z)$ as a function of $z$, with $\lambda=2^{k-1}$ :

$$
\left|v_{k}(x)\right|^{2} \lesssim 2^{-k} \int_{|z|<2^{-k+1}}\left|\Delta_{z} v_{k}(x+z)\right|^{2} d z+2^{3 k} \int_{|z|<2^{-k+1}}\left|v_{k}(x+z)\right|^{2} d z .
$$

Taking advantage of $\Delta_{z} v_{k}(x+z)=\Delta_{x} v_{k}(x+z)$, we can combine (21) for $v_{k}$ with (21) where $v_{k}$ is replaced by $\Delta v_{k}$ which is also a solution to the Schrödinger equation with Dirichlet boundary condition:

$$
\begin{aligned}
\int_{0}^{T} \int_{\Omega}\left|v_{k}(x)(\omega \cdot \nabla) u_{j}(x)\right|^{2} d x d t & \lesssim 2^{-k} K_{\omega}\left(u, \Delta v_{k}\right)+2^{3 k} K_{\omega}\left(u, v_{k}\right) \\
\lesssim & 2^{-2 k}\left(\left\|\Delta v_{k}(0)\right\|_{2}^{2} \mathcal{H}_{0}\left(u_{j}\right)+\left\|u_{j}(0)\right\|_{2}^{2} \mathcal{H}_{0}\left(\Delta v_{k}\right)\right) \\
& +2^{2 k}\left(\left\|v_{k}(0)\right\|_{2}^{2} \mathcal{H}_{0}\left(u_{j}\right)+\left\|u_{j}(0)\right\|_{2}^{2} \mathcal{H}_{0}\left(v_{k}\right)\right) \\
\lesssim & 2^{2 k}\left(\left\|v_{k}(0)\right\|_{2}^{2} \mathcal{H}_{0}\left(u_{j}\right)+\left\|u_{j}(0)\right\|_{2}^{2} \mathcal{H}_{2}\left(v_{k}\right)\right)
\end{aligned}
$$

Which is the desired conclusion, as $\omega$ is any direction in $\mathbb{S}^{2}$. Theorem 2.1 is proved.

Notice that

$$
\int_{0}^{T}\left\|u_{j} \nabla v_{k}\right\|_{2}^{2} d t \lesssim\left(\int_{0}^{T}\left\|u_{j}\right\|_{4}^{4} d t\right)^{\frac{1}{2}}\left(\int_{0}^{T}\left\|\sqrt{-\Delta} v_{k}\right\|_{4}^{4} d t\right)^{\frac{1}{2}} \lesssim\left\|u_{j}(0)\right\|_{2} \mathcal{H}_{0}\left(u_{j}\right)^{\frac{1}{2}}\left\|v_{k}(0)\right\|_{2} \mathcal{H}_{0}\left(v_{k}\right)^{\frac{1}{2}}
$$

so that we also control $\nabla\left(u_{j} v_{k}\right)$ or $\nabla\left(u_{j} \bar{v}_{k}\right)$ rather than just $v_{k} \nabla u_{j}$. This will prove to be useful in the next argument.

We now prove (5) from Proposition 2.1 and (6) from Proposition 2.2. let us sumarize our current result as

$$
2^{-2 k-j} \int_{0}^{T}\left\|u_{j} \nabla v_{k}\right\|_{2}^{2}+\left\|\nabla\left(u_{j} v_{k}\right)\right\|_{2}^{2} d t \lesssim 2^{-k} \mathcal{H}_{2}\left(v_{k}\right)\left\|u_{j}(0)\right\|_{2}^{2}+2^{-j} \mathcal{H}_{0}\left(u_{j}\right)\left\|v_{k}(0)\right\|_{2}^{2}=\Gamma\left(u_{j}, v_{k}\right) .
$$

Let $\Delta_{l}=\varphi\left(2^{-2 l} \Delta\right)$ be a spectral localization on $\Omega$. Let $Q_{l}=2^{-l} \nabla \exp \left(2^{-2 l} \Delta\right)$ and $Q_{l}^{\star}$ its transpose. We need to prove

$$
\int_{0}^{T}\left\|u_{j} v_{k}\right\|_{2}^{2} d t \approx \sum_{l} \int_{0}^{T}\left\|\Delta_{l}\left(u_{j} v_{k}\right)\right\|_{2}^{2} d t \lesssim 2^{2 k-j}\left\|u_{j}(0)\right\|_{2}^{2}\left\|v_{k}(0)\right\|_{2}^{2},
$$

where $T$ is arbitrarily large on a non-trapping domain or $T<2^{-j}$ on a bounded domain. 
Start with $l>j$ : we have

$$
\Delta_{l}\left(u_{j} v_{k}\right)=\tilde{\Delta}_{l} \exp \left(2^{-2 l} \Delta\right) 2^{-2 l} \Delta\left(u_{j} v_{k}\right)=\tilde{\Delta}_{l} Q_{l}^{\star} 2^{-l} \nabla\left(u_{j} v_{k}\right)
$$

where $\tilde{\Delta}_{l}=\psi\left(2^{-2 l} \Delta\right)$ and $\psi(\xi)=\varphi(\xi) \xi^{-1} \exp (\xi) \in C_{0}^{\infty}\left(\mathbb{R}_{+}^{*}\right)$, and therefore, as $\tilde{\Delta}_{j}, Q_{l}^{\star}$ are bounded on $L^{2}$ (see [21])

$$
\int_{0}^{T}\left\|\Delta_{l}\left(u_{j} v_{k}\right)\right\|_{2}^{2} d t \lesssim 2^{-2 l+j+2 k} \Gamma\left(u_{j}, v_{k}\right)
$$

which we may sum over $l>j$ to get that

$$
\sum_{l>j} \int_{0}^{T}\left\|\Delta_{l}\left(u_{j} v_{k}\right)\right\|_{2}^{2} d t \lesssim 2^{-j+2 k} \Gamma\left(u_{j}, v_{k}\right) .
$$

We now proceed with $l<j$. We consider directly

$$
\sum_{l<j} \Delta_{l}\left(u_{j} v_{k}\right)=S_{j}\left(u_{j} v_{k}\right)
$$

Recall $u_{j}=\phi\left(2^{-2 j} \Delta\right) u_{j}$ where $\phi$ is compactly supported away from $\xi=0$. Let $\tilde{\phi} \in C_{0}^{\infty}\left(\mathbb{R}_{+}^{*}\right)$ be such that $\xi^{-1} \tilde{\phi}(\xi)=1$ on the support of $\phi$, and $\mathcal{D}_{j}=\tilde{\phi}\left(2^{-2 j} \Delta\right)$. We may rewrite

$$
S_{j}\left(u_{j} v_{k}\right)=S_{j}\left(v_{k} 2^{-2 j} \Delta \mathcal{D}_{j} u_{j}\right),
$$

and $w_{j}=\mathcal{D}_{j} u_{j}$ is a solution to (2) with data $w_{j}(0)=\mathcal{D}_{j} u_{j}(0)$. Then

$$
\begin{aligned}
S_{j}\left(u_{j} v_{k}\right) & =S_{j}\left(2^{-j} \operatorname{div}\left(v_{k} 2^{-j} \nabla w_{j}\right)-S_{j}\left(2^{-j} \nabla v_{k} \cdot 2^{-j} \nabla w_{j}\right)\right. \\
& =\tilde{S}_{j} Q_{j}^{\star}\left(v_{k} 2^{-j} \nabla w_{j}\right)-2^{k-j} S_{j}\left(2^{-k} \nabla v_{k} \cdot 2^{-j} \nabla w_{j}\right),
\end{aligned}
$$

where $\tilde{S}_{j}=\gamma\left(2^{-2 j} \Delta\right)$ and $\gamma(\xi)=\sum_{l<0} \varphi\left(2^{-2 l} \xi\right) \exp (\xi) \in C_{0}^{\infty}([0,+\infty))$. Again, $S_{j}, \tilde{S}_{j}, Q_{j}^{\star}$ are bounded on $L^{2}$ and therefore, using (24) on $v_{k} \nabla w_{j}$ and Lemma 4.1 for $\nabla v_{k}$ and $\nabla w_{j}$,

$$
\begin{aligned}
\int_{0}^{T}\left\|S_{j}\left(u_{j} v_{k}\right)\right\|_{2}^{2} d t & \lesssim 2^{-j} 2^{2 k+j} \Gamma\left(w_{j}, v_{k}\right)+2^{2(k-j)}\left\|w_{j}(0)\right\|_{2} \mathcal{H}_{0}\left(w_{j}\right)^{\frac{1}{2}}\left\|v_{k}(0)\right\|_{2} \mathcal{H}_{0}\left(v_{k}\right)^{\frac{1}{2}} \\
& \lesssim 2^{2 k-j} \Gamma\left(w_{j}, v_{k}\right)+2^{2(k-j)} 2^{j / 2+k / 2} \Gamma\left(w_{j}, v_{k}\right),
\end{aligned}
$$

and using $k<j$ and continuity of $\mathcal{D}_{j}$,

$$
\int_{0}^{T}\left\|S_{j}\left(u_{j} v_{k}\right)\right\|_{2}^{2} d t \lesssim 2^{2 k-j} \Gamma\left(w_{j}, v_{k}\right) .
$$

In order to complete the proof, we proceed differently depending on the domain. Let $S$ be a (compact) strip close to the boundary $\partial \Omega$, we recall the following estimate from [28] (eq. (5.4) p. 279 ), on solutions to (1) with $\varepsilon=0$ (linear equation):

$$
\int_{0}^{T} \int_{\partial \Omega}\left|\partial_{n} \phi\right|^{2} d S d t \lesssim \int_{0}^{T}\|\phi\|_{H^{1}(S)}^{2} d t+\sup _{[0, T]}\|\phi\|_{\dot{H}^{1 / 2}}^{2} .
$$

We are now facing two different situations:

- if $\Omega$ is the exterior of a non-trapping compact obstacle, local smoothing holds ([8]) and the time integral on the right-hand side of (27) is controlled by the other term, itself controlled by invariants of the flow. Hence,

$$
\int_{0}^{T} \int_{\partial \Omega}\left|\partial_{n} \phi\right|^{2} d S d t \lesssim\|\phi\|_{2}\|\phi\|_{H_{0}^{1}(\Omega)} .
$$

Recalling the definition of $\Gamma(\cdot, \cdot), \mathcal{H}_{k}(\cdot)$ and using the frequencies localizations, we get

$$
\Gamma\left(w_{j}, v_{k}\right) \lesssim\left\|w_{j}(0)\right\|_{2}\left\|v_{k}(0)\right\|_{2} \lesssim\left\|u_{j}(0)\right\|_{2}\left\|v_{k}(0)\right\|_{2} .
$$


Gathering (25), (26) we obtain the desired estimate (5);

- if $\Omega$ is a compact domain, we simply restrict $T<2^{-j}$, so that

$$
\int_{0}^{T} \int_{\partial \Omega}\left|\partial_{n} \phi\right|^{2} d S d t \lesssim 2^{-j}\|\phi(0)\|_{H_{0}^{1}(\Omega)}^{2}+\|\phi(0)\|_{2}\|\phi(0)\|_{H_{0}^{1}(\Omega)} .
$$

Now, we get again

$$
\Gamma\left(w_{j}, v_{k}\right) \lesssim\left\|w_{j}(0)\right\|_{2}\left\|v_{k}(0)\right\|_{2} \lesssim\left\|u_{j}(0)\right\|_{2}\left\|v_{k}(0)\right\|_{2},
$$

but only for $T<2^{-j}$. Gathering (25), (26) we obtain the desired estimate (6); for any interval $[0, T]$, we split into intervals of size $2^{-j}$ and sum up, which yields (7).

This achieves the proof of Propositions 2.1 and 2.2 .

4.1. Nonlinear equation on a domain. We shall use the following result to estimate norms in our conormal spaces $X_{(l)}^{1}$ and $X^{s}$, which can be extracted from [17].

Lemma 4.3. Consider $u$ the solution of $i \partial_{t}+\Delta u=f, u_{\mid \partial \Omega}=0 u_{\mid t=0}=u_{0}$. Then for any $s$,

$$
\|u\|_{X^{s}} \leqslant C_{T}\left(\left\|u_{0}\right\|_{B_{2}^{s, 2}}+\|f\|_{\left(Y^{-s}\right)^{\prime}}\right),
$$

where $\left(Y^{-s}\right)^{\prime}$ is the dual space (with respect to the $L_{t, x}^{2}$ bracket) of $Y^{s}$.

Remark 4.1. It should be emphasized that, for small $T, C_{T} \sim C$, which is a consequence of our use of the $U$ and $V$ spaces. Hence, in a contraction argument, the smallness may not be provided by the Duhamel estimate (as is customary with the classical $X^{s, b}$ spaces), nor by rescaling since we are on a domain. However, the nonlinear estimate will be derived using (77) where the $T$ factor will serve this purpose.

The important feature of the $U$ and $V$ based spaces is that we have a good transference principle: multilinear estimates involving linear solutions to the Schrödinger equation may be turned into estimates for our $X_{(l)}^{s}$ spaces. From now on, it should be understood that we restrict the time interval to a fixed time interval $[-T, T]$. Given that functions in our spaces can be time-truncated this is harmless.

Proposition 4.1. Let $u^{(1)}, u^{(2)}, u^{(3)} \in X_{l}^{1}$ then $u^{(1)} \bar{u}^{(2)} u^{(3)} \in\left(Y_{l}^{-1}\right)^{\prime}$ and

$$
\left\|u^{(1)} \bar{u}^{(2)} u^{(3)}\right\|_{\left(Y_{l}^{-1}\right)^{\prime}} \lesssim T\left\|u^{(1)}\right\|_{X_{l}^{1}}\left\|u^{(2)}\right\|_{X_{l}^{1}}\left\|u^{(3)}\right\|_{X_{l}^{1}} .
$$

Moreover, if $u \in X^{s}$, for $1<s \leqslant 3$, then

$$
\left\||u|^{2} u\right\|_{\left(Y^{-s}\right)^{\prime}} \lesssim T\|u\|_{X_{l}^{1}}^{2}\|u\|_{X^{s}} .
$$

Both nonlinear mappings are proved by duality: for convenience, introduce $v \in Y_{l}^{1}$, then (30) is a consequence of

$$
n\left|\int_{0}^{T} \int_{\Omega} u_{1} \bar{u}_{2} u_{3} \Delta \bar{v} d x d t\right| \lesssim T\left\|u^{(1)}\right\|_{X_{l}^{1}}\left\|u^{(2)}\right\|_{X_{l}^{1}}\left\|u^{(3)}\right\|_{X_{l}^{1}}\|v\|_{Y_{l}^{1}},
$$

while, with $v \in Y^{2-s}$, (31) is a consequence of

$$
\left|\int_{0}^{T} \int_{\Omega} u \bar{u} u \Delta \bar{v} d x d t\right| \lesssim T\|u\|_{X_{l}^{1}}^{2}\|u\|_{X^{s}}\|v\|_{Y^{2-s}} .
$$

Both space-time integrals may now be decomposed into dyadic pieces, and we are left with estimating

$$
I_{j_{1}, j_{2}, j_{3}, j_{4}}=\int_{0}^{T} \int_{\Omega} u_{j_{1}}^{(1)} \bar{u}_{j_{2}}^{(2)} u_{j_{3}}^{(3)} \Delta v_{j_{4}} d x d t
$$

where the first three factors are dyadic pieces of the corresponding $u^{(1,2,3)}$ and the fourth one of $v$. In order to proceed, we recall the following end-point result, which will be crucial: 
Proposition $4.2([3])$. The solution $w_{m}$ to the linear Schrödinger equation (2) satisfies

$$
\left\|w_{m}\right\|_{L_{T}^{2} L^{\infty}(\Omega)} \lesssim \sqrt{T} m^{2} 2^{m}\left\|w_{m}(0)\right\|_{2} .
$$

This is nothing but a summation over time intervals of [3], Lemma 6.1 (which is stated on an interval of size $2^{-m}$ ), together with conservation of mass. Notice that (33) has a double logarithmic loss in the frequency. We may now use Proposition 2.19 (i) in [17] to obtain, for a generic $w_{m}$ which is frequency localized at $2^{m}$,

$$
\left\|w_{m}\right\|_{L_{T}^{2} L^{\infty}(\Omega)} \lesssim \sqrt{T} m^{2} 2^{m}\left\|w_{m}\right\|_{U_{S}^{2}},
$$

while we have the obvious "energy" estimate $\left\|w_{m}\right\|_{L_{t}^{\infty} L^{2}} \lesssim\left\|w_{m}\right\|_{U_{S}^{q}}$ for any $q \leqslant+\infty$. We may now state our key estimate as a suitable analog of Proposition 2.2 .

Proposition 4.3. Let $u_{j}$ and $v_{k}$ be frequency localized functions with $k \leqslant j$, then

$$
\int_{0}^{T}\left\|u_{j} v_{k}\right\|_{2}^{2} d t+2^{-2 j} \int_{0}^{T}\left\|v_{k} \nabla u_{j}\right\|_{2}^{2} d t \lesssim T 2^{2 k}\left\|u_{j}\right\|_{U_{S}^{2}}^{2}\left\|v_{k}(0)\right\|_{U_{S}^{2}}^{2}
$$

and

$$
\int_{0}^{T}\left\|u_{j} v_{k}\right\|_{2}^{2} d t+2^{-2 j} \int_{0}^{T}\left\|v_{k} \nabla u_{j}\right\|_{2}^{2} d t \lesssim T 2^{2 k}(\log k)^{2}\left\|u_{j}\right\|_{V_{S}^{2}}^{2}\left\|v_{k}(0)\right\|_{U_{S}^{2}}^{2}
$$

Moreover,

$$
2^{-2 k-2 j} \int_{0}^{T}\left\|u_{j} \Delta v_{k}\right\|_{2}^{2}+2^{-2 j} \int_{0}^{T}\left\|u_{j} \nabla v_{k}\right\|_{2}^{2} d t \lesssim T 2^{3 k-j}\left\|u_{j}\right\|_{V_{S}^{2}}^{2}\left\|v_{k}(0)\right\|_{V_{S}^{2}}^{2} .
$$

Proof. The first estimate (35) is a direct consequence of (7) and, again, Proposition 2.19 (i) in [17], which is the transference principle we alluded to. The third estimate (37) is a consequence of a linear estimate: again by Proposition 2.19 in [17], we have, as a consequence of (19), that for any $w_{m}$ localized at $2^{m}, l \in \mathbb{N}$,

$$
\left\|(\nabla)^{l} w_{m}\right\|_{L_{T}^{4} L^{4}} \lesssim T^{\frac{1}{4}} 2^{\left(\frac{1}{2}+l\right) m}\left\|w_{m}\right\|_{U_{S}^{4}} \lesssim T^{\frac{1}{4}} 2^{\left(\frac{1}{2}+l\right) m}\left\|w_{m}\right\|_{V_{S}^{2}},
$$

using $U_{S}^{4} \subset V_{S}^{2}$. Hence (37) follows by Hölder. The second (key !) estimate (36) is proven in two steps: first, combining by Hölder (34) on $v_{k}$ and the trivial "energy" estimate on $u_{j}$, we get a new bilinear estimate,

$$
2^{-2 j} \int_{0}^{T}\left\|v_{k} \nabla u_{j}\right\|_{2}^{2} d t+\int_{0}^{T}\left\|u_{j} v_{k}\right\|_{2}^{2} d t \lesssim T 2^{2 k} k^{4}\left\|u_{j}\right\|_{U_{S}^{\infty}}^{2}\left\|v_{k}(0)\right\|_{U_{S}^{2}}^{2} .
$$

We may now apply Proposition 2.20 from [17] to the operator $\mathcal{T}$ mapping $u_{j}$ to $u_{j} v_{k}$ : by (39) it maps $U_{S}^{\infty}$ to $L_{T}^{2} L^{2}$ with constant $C_{1} \sqrt{T} 2^{k} k^{2}\left\|v_{k}(0)\right\|_{U_{S}^{2}}$, while by (35) it maps $U_{S}^{2}$ to $L_{T}^{2} L^{2}$ with constant $C_{2} \sqrt{T} 2^{k}\left\|v_{k}(0)\right\|_{U_{S}^{2}}$. As a result, $\mathcal{T}$ maps $V_{S}^{2}$ to $L_{T}^{2} L^{2}$ with constant $C_{3} \sqrt{T} 2^{k} \log k\left\|v_{k}(0)\right\|_{U_{S}^{2}}$ which is the desired result. The estimate for $v_{k} \nabla u_{j}$ follows similarly.

We remark that the right-hand side of (37) may obviously be replaced by the right-hand side of (36)), as $U_{S}^{2} \subset V_{S}^{2}$. This will be useful in unifying the treatment of nonlinear terms later.

Lemma 4.4. Let $j_{1}, j_{2}, j_{4} \leqslant j_{3}$, we have

$$
\sum_{j_{1}, j_{2}}\left|I_{j_{1}, j_{2}, j_{3}, j_{4}}\right| \lesssim T\left\|u^{(1)}\right\|_{X_{l}^{1}}\left\|u^{(2)}\right\|_{X_{l}^{1}}\left\|u_{j_{3}}^{(3)}\right\|_{U_{S}^{2}}\left\|\Delta v_{j_{4}}\right\|_{V_{S}^{2}} .
$$


Proof. One should think that the most difficult case is when $j_{1}, j_{2} \leqslant j_{4}$ : Estimate (40) then follows from (35) and (36) after pairing both low frequency factors with the higher frequencies factors, applying Cauchy-Schwarz, summing over $j_{1}, j_{2}$ and using the definition of $X_{l}^{1}$ : without loss of generality, let $j_{1} \leqslant j_{2}$,

$$
\begin{aligned}
\left|I_{j_{1}, j_{2}, j_{3}, j_{4}}\right| & \lesssim\left\|u_{j_{2}}^{(2)} u_{j_{3}}^{(3)}\right\|_{L_{T}^{2} L^{2}}\left\|u_{j_{1}}^{(1)} \Delta v_{j_{4}}\right\|_{L_{T}^{2} L^{2}} \\
& \lesssim T \log j_{1} 2^{j_{1}}\left\|u_{j_{1}}^{(1)}\right\|_{U_{S}^{2}} 2^{j_{2}}\left\|u_{j_{2}}^{(2)}\right\|_{U_{S}^{2}}\left\|u_{j_{3}}^{(3)}\right\|_{U_{S}^{2}}\left\|\Delta v_{j_{4}}\right\|_{V_{S}^{2}} \\
\sum_{j_{1} \leqslant j_{2}}\left|I_{j_{1}, j_{2}, j_{3}, j_{4}}\right| & \lesssim \sum_{j_{1} \leqslant j_{2}} T\left(\log j_{1}\right)^{\frac{1}{2}} 2^{j_{1}}\left\|u_{j_{1}}^{(1)}\right\|_{U_{S}^{2}}\left(\log j_{2}\right)^{\frac{1}{2}} 2^{j_{2}}\left\|u_{j_{2}}^{(2)}\right\|_{U_{S}^{2}}\left\|u_{j_{3}}^{(3)}\right\|_{U_{S}^{2}}\left\|\Delta v_{j_{4}}\right\|_{V_{S}^{2}}
\end{aligned}
$$

which implies the desired result. When $j_{4}$ is less than $j_{1}$ and/or $j_{2}$, we simply use (37) on the $v$ factor and one of the $u$ 's.

Assume that $\epsilon=0,1 / 2, s>0,1 \leqslant p$ together with

$$
2^{s j_{3}}\left(\log j_{3}\right)^{\epsilon}\left\|u_{j_{3}}^{(3)}\right\|_{U_{S}^{2}}=\alpha_{j_{3}} \in l^{p} \text { and } 2^{(2-s) j_{4}}\left(\log j_{4}\right)^{-\epsilon}\left\|v_{j_{4}}\right\|_{U_{S}^{2}}=\beta_{j_{4}} \in l^{p^{\prime}}
$$

then we may sum over $j_{3} \leqslant j_{4}$ (using Young for discrete sequences) to get

$$
\sum_{j_{1} \leqslant j_{2} \leqslant j_{4} \leqslant j_{3}}\left|I_{j_{1}, j_{2}, j_{3}, j_{4}}\right| \lesssim\left\|u^{(1)}\right\|_{X_{l}^{1}}\left\|u^{(2)}\right\|_{X_{l}^{1}}\left\|\left(\alpha_{j_{3}}\right)_{j_{3}}\right\|_{l^{p}}\left\|\left(\beta_{j_{4}}\right)_{j_{4}}\right\|_{l^{p^{\prime}}}
$$

Both nonlinear mappings from Proposition 4.1 follow for such a restricted set of indices $\left\{j_{1}, j_{2}, j_{4} \leqslant\right.$ $\left.j_{3}\right\}$, choosing $s=1, p=1, \epsilon=1 / 2$ and then $1<s \leqslant 3, p=2, \epsilon=0$.

We now deal with the most difficult situation, namely $j_{1}, j_{2}, j_{3} \leqslant j_{4}$. Without loss of generality, we may suppose $j_{1} \leqslant j_{2} \leqslant j_{3}$. Integrating by parts the full Laplacian, we get terms like

$$
J_{j_{1}, j_{2}, j_{3}, j_{4}}=\int_{0}^{T} \int_{\Omega} u_{j_{1}}^{(1)} \bar{u}_{j_{2}}^{(2)} \Delta u_{j_{3}}^{(3)} v_{j_{4}} d x d t \text { and } K_{j_{1}, j_{2}, j_{3}, j_{4}}=\int_{0}^{T} \int_{\Omega} u_{j_{1}}^{(1)} \nabla \bar{u}_{j_{2}}^{(2)} \cdot \nabla u_{j_{3}}^{(3)} v_{j_{4}} d x d t
$$

where successive boundary terms vanish due to the Dirichlet boundary condition and where we kept only the most difficult terms (derivatives fall on the highest frequencies).

Lemma 4.5. Let $j_{1}, j_{2} \leqslant j_{3} \leqslant j_{4}$, we have

$$
\sum_{j_{1}, j_{2}}\left|J_{j_{1}, j_{2}, j_{3}, j_{4}}\right| \lesssim T\left\|u^{(1)}\right\|_{X_{l}^{1}}\left\|u^{(2)}\right\|_{X_{l}^{1}}\left\|\Delta u_{j_{3}}^{(3)}\right\|_{U_{S}^{2}}\left\|v_{j_{4}}\right\|_{V_{S}^{2}}
$$

while

$$
\sum_{j_{1}, j_{2} \leqslant j_{3}}\left|K_{j_{1}, j_{2}, j_{3}, j_{4}}\right| \lesssim T\left\|u^{(1)}\right\|_{X_{l}^{1}}\left\|u^{(2)}\right\|_{X_{l}^{1}} 2^{\frac{3}{2} j_{3}}\left\|\Delta u_{j_{3}}^{(3)}\right\|_{U_{S}^{2}} 2^{\frac{1}{2} j_{4}}\left\|v_{j_{4}}\right\|_{V_{S}^{2}}
$$

Proof. The $J_{j_{1}, j_{2}, j_{3}, j_{4}}$ integral may be dealt with like we did for $I_{j_{1}, j_{2}, j_{3}, j_{4}}$ : indeed,

$$
\begin{aligned}
\left|J_{j_{1}, j_{2}, j_{3}, j_{4}}\right| & \lesssim\left\|u_{j_{2}}^{(2)} \Delta u_{j_{3}}^{(3)}\right\|_{L_{T}^{2} L^{2}}\left\|u_{j_{1}}^{(1)} v_{j_{4}}\right\|_{L_{T}^{2} L^{2}} \\
\sum_{j_{1} \leqslant j_{2}}\left|I_{j_{1}, j_{2}, j_{3}, j_{4}}\right| & \lesssim \sum_{j_{1} \leqslant j_{2}} T\left(\log j_{1}\right)^{\frac{1}{2}} 2^{j_{1}}\left\|u_{j_{1}}^{(1)}\right\|_{U_{S}^{2}}\left(\log j_{2}\right)^{\frac{1}{2}} 2^{j_{2}}\left\|u_{j_{2}}^{(2)}\right\|_{U_{S}^{2}}\left\|\Delta u_{j_{3}}^{(3)}\right\|_{U_{S}^{2}}\left\|v_{j_{4}}\right\|_{V_{S}^{2}} \\
\sum_{j_{1} \leqslant j_{2}}\left|J_{j_{1}, j_{2}, j_{3}, j_{4}}\right| & \lesssim\left\|u^{(1)}\right\|_{X_{l}^{1}}\left\|u^{(2)}\right\|_{X_{l}^{1}}\left\|\Delta u_{j_{3}}^{(3)}\right\|_{U_{S}^{2}}\left\|v_{j_{4}}\right\|_{V_{S}^{2}}
\end{aligned}
$$


The $K_{j_{1}, j_{2}, j_{3}, j_{4}}$ is interesting in that it does not require the use of (36) but only (35) and the linear $L_{t x}^{4}$ estimate (38):

$$
\begin{aligned}
\left|K_{j_{1}, j_{2}, j_{3}, j_{4}}\right| & \lesssim\left\|u_{j_{1}}^{(1)} \nabla u_{j_{3}}^{(3)}\right\|_{L_{T}^{2} L^{2}}\left\|\nabla u_{j_{2}}^{(2)}\right\|_{L_{T}^{4} L^{4}}\left\|v_{j_{4}}\right\|_{L_{T}^{4} L^{4}} \\
\sum_{j_{1} \leqslant j_{2}}\left|I_{j_{1}, j_{2}, j_{3}, j_{4}}\right| & \lesssim \sum_{j_{1} \leqslant j_{2}} T 2^{j_{1}}\left\|u_{j_{1}}^{(1)}\right\|_{U_{S}^{2}} 2^{\frac{3}{2} j_{2}}\left\|u_{j_{2}}^{(2)}\right\|_{U_{S}^{4}} 2^{j_{3}}\left\|u_{j_{3}}^{(3)}\right\|_{U_{S}^{2}} 2^{\frac{1}{2} j_{4}}\left\|v_{j_{4}}\right\|_{V_{S}^{2}} \\
\sum_{j_{1} \leqslant j_{2} \leqslant j_{3}}\left|J_{j_{1}, j_{2}, j_{3}, j_{4}}\right| & \lesssim\left\|u^{(1)}\right\|_{X_{l}^{1}}\left\|u^{(2)}\right\|_{X_{l}^{1}} 2^{\frac{3}{2} j_{3}}\left\|\Delta u_{j_{3}}^{(3)}\right\|_{U_{S}^{2}} 2^{\frac{1}{2} j_{4}}\left\|v_{j_{4}}\right\|_{V_{S}^{2}}
\end{aligned}
$$

where we notice that the $\log$ factor in $X_{l}^{1}$ could be dispensed with.

Now, we may again conclude as we did before for both remaining sums over $j_{3} \leqslant j_{4}$, in order to achieve the proof of Proposition 4.1, for $1<s<2$.

However, for $2 \leqslant s \leqslant 3$, we need to integrate by parts again: in fact, $v \in Y^{2-s}$ is still at zero or negative regularity, so we set $v=\Delta w$ and we consider

$$
\tilde{J}_{j_{1}, j_{2}, j_{3}, j_{4}}=\int_{0}^{T} \int_{\Omega} u_{j_{1}}^{(1)} \bar{u}_{j_{2}}^{(2)} \Delta u_{j_{3}}^{(3)} \Delta w_{j_{4}} d x d t \text { and } \tilde{K}_{j_{1}, j_{2}, j_{3}, j_{4}}=\int_{0}^{T} \int_{\Omega} u_{j_{1}}^{(1)} \nabla \bar{u}_{j_{2}}^{(2)} \cdot \nabla u_{j_{3}}^{(3)} \Delta w_{j_{4}} d x d t
$$

where, again, we kept what is, at this stage, the worst distribution of derivatives. The $\tilde{J}_{j_{1}, j_{2}, j_{3}, j_{4}}$ integral is nothing but our original $I_{j_{1}, j_{2}, j_{3}, j_{4}}$ is disguise, with $\Delta u_{j_{3}}$ playing the part of $u_{j_{3}}$ and $w$ the part of $v$; we leave the details to the reader. The $\tilde{K}_{j_{1}, j_{2}, j_{3}, j_{4}}$ integral requires more care: first, remark that we may integrate by parts twice, as the successive boundary terms will vanish due to $u_{j_{1} \mid \partial \Omega}=0$ and then $w_{\partial \Omega}=0$. If the Laplacian hits any individual $u_{j}$ factor, we are back with an integral like our previous $K_{j_{1}, j_{2}, j_{3}, j_{4}}$. Hence the last remaining case to deal with corresponds to

$$
L_{j_{1}, j_{2}, j_{3}, j_{4}}=\int_{0}^{T} \int_{\Omega} u_{j_{1}}^{(1)} \nabla^{2} \bar{u}_{j_{2}}^{(2)} \nabla^{2} u_{j_{3}}^{(3)} w_{j_{4}} d x d t
$$

for which we use (36) on the $u_{j_{1}} w_{j_{4}}$ factor and the linear estimate (38) for the remaining two factors.

Remark 4.2. One should emphasize that once a global solution is obtained for $1<s<\frac{3}{2}$ (so that $\left.H_{0}^{s}=H^{s}(\Omega) \cap H_{0}^{1}\right)$, using the available Strichartz estimates one could propagate classical $H^{\sigma}(\Omega)$ regularity for $\sigma>s$ by using the equation and energy estimates. The main point of the previous analysis in $X^{s}$ spaces is to illustrate that one may perform more than one integration by parts in the analysis of the worst case scenario $j_{4}>j_{3}$ (which does not exist in $\mathbb{R}^{3}$ by orthogonality due to disjoint Fourier supports). In fact, one could perform one last integration by parts (with just one gradient) in the worst part of $\tilde{J}_{j_{1}, j_{2}, j_{3}, j_{4}}$ to reach $s<4$, taking advantage of $\partial_{n}^{2} u_{\mid \partial \Omega}=0$ to dispense with the corresponding boundary term.

Let us denote by \|\|$u\left\|_{T}=\right\| \mathbf{1}_{(-T, T)} u \|_{X_{l}^{1}}$. Proposition 3.1 is a simple consequence of Banach fixed-point theorem in a suitable ball of the space endowed with norm $\|\cdot \mid \cdot\|_{T}$. From now on, all numerical constants are written explicitly and numbered. We have

$$
\||u|\|_{T} \leqslant\left\|S(t) u(0)\left|\left\|_{T}+C_{1} T\right\| u\right|\right\|_{T}^{3} \leqslant C_{0}\|u(0)\|_{B_{2, l}^{1,1}}+C_{1} T\|\| u \|_{T}^{3} .
$$

And we may solve for

$$
\frac{C_{2}}{2\|u(0)\|_{B_{2 l}^{1,1}}^{2}} \leqslant T<\frac{C_{2}}{\|u(0)\|_{B_{2, l}^{1,1}}^{2}} .
$$


We now proceed with Proposition 3.2, we only need to prove an estimate on the time of existence, persistence in $X^{s}$ being a direct consequence of (31), which we may rewrite, with $\|u\|_{s, T}=$ $\left\|\mathbf{1}_{(-T, T)} u\right\|_{X^{s}}$,

$$
\|u\|_{s, T} \leqslant C_{3}\|u(0)\|_{H^{s}}+C_{4} T\|u\|_{T}^{2}\|u\|_{s, T} .
$$

As such, adjusting the $C_{2}$ constant if necessary,

$$
\|u(T)\|_{H^{s}} \leqslant\|u\|_{s, T} \leqslant 2 C_{3}\|u(0)\|_{H^{s}}
$$

provided $T$ satisfies (45). We now proceed with a logarithmic Sobolev inequality: for any $f \in B_{2, l}^{1,1}$, for $s=1+\eta$, we have, for $J$ large,

$$
\begin{aligned}
\|f\|_{B_{2, l}^{1,1}} & \leqslant\left\|S_{0} f\right\|_{2}+\sum_{j \geqslant 0}^{J}(\log j)^{\frac{1}{2}} 2^{j}\left\|\Delta_{j} f\right\|_{2}+\sum_{j \geqslant J}(\log j)^{\frac{1}{2}} 2^{j}\left\|\Delta_{j} f\right\|_{2} \\
& \leqslant\left\|S_{0} f\right\|_{2}+(J \log J)^{\frac{1}{2}}\left(\sum_{j \geqslant 0}^{J} 2^{2 j}\left\|\Delta_{j} f\right\|_{2}^{2}\right)^{\frac{1}{2}}+\sum_{j \geqslant J}(\log j)^{\frac{1}{2}} 2^{-\eta j} 2^{s j}\left\|\Delta_{j} f\right\|_{2} \\
& \leqslant\|f\|_{2}+(J \log J)^{\frac{1}{2}}\|f\|_{H^{1}}+2^{-\frac{3}{4} \eta J}\|f\|_{H^{s}} \\
& \leqslant\|f\|_{2}+(J \log J)^{\frac{1}{2}}\left(\|f\|_{H^{1}}+2^{-\frac{\eta}{2} J}\|f\|_{H^{s}}\right)
\end{aligned}
$$

and the $(\cdots)$ is optimized in $J$ for $J=2 / \eta \log \left(\eta\|f\|_{H^{s}} /\left(2\|f\|_{H^{1}}\right)\right)$. As such, we get

$$
\|f\|_{B_{2}^{1,1}} \leqslant\|f\|_{H^{1}}\left(C_{5}+C_{6}\left(\log \|f\|_{H^{s}} \log \log \|f\|_{H^{s}}\right)^{\frac{1}{2}}\right) .
$$

Using this inequality, for $H^{s}$ data, (45) yields a new time of existence,

$$
T \sim \frac{C_{7}}{\|u(0)\|_{H^{1}}^{2} \log \|u(0)\|_{H^{s}} \log \log \|u(0)\|_{H^{s}}},
$$

and the proof of Proposition 3.2 is complete.

Finally, we prove Theorem 3.1 by iterating the local in time construction from Proposition 3.2. In the defocusing case of (11), the Hamiltonian $E(u)$ is conserved and controls the $H^{1}$ norm. Set $T_{1}=T$, and repeat the local in time construction from the data $u\left(T_{1}\right)$, and reach $T_{1}+T_{2}$, with

$$
T_{2} \sim \frac{C_{7}}{E \log \left\|u\left(T_{1}\right)\right\|_{H^{s}} \log \log \left\|u\left(T_{1}\right)\right\|_{H^{s}}} \sim \frac{C_{7}}{E \log \left(2 C_{3}\|u(0)\|_{H^{s}}\right) \log \log \left(2 C_{3}\|u(0)\|_{H^{s}}\right)} .
$$

after $n$ steps, we reach $T_{1}+T_{2}+\cdots+T_{n}$, with

$$
T_{n} \sim \frac{C_{7}}{E \log \left\|u\left(T_{n-1}\right)\right\|_{H^{s}} \log \log \left\|u\left(T_{n-1}\right)\right\|_{H^{s}}} \sim \frac{C_{7}}{E \log \left(\left(2 C_{3}\right)^{n-1}\|u(0)\|_{H^{s}}\right) \log \log \left(\left(2 C_{3}\right)^{n-1}\|u(0)\|_{H^{s}}\right)}
$$

which yields for $n$ large enough (depending on $C_{3}$ and $\|u(0)\|_{H^{s}}$,

$$
T_{n} \geqslant \frac{C_{8}}{n \log n} .
$$

Therefore the series $\sum_{n} T_{n}$ diverges and we may reach an arbitrarily large time. Moreover,

$$
\left\|u\left(T_{n}\right)\right\|_{H^{s}} \leqslant\left(2 C_{3}\right)^{n-1}\|u(0)\|_{H^{s}} \sim C_{9}\left(\exp \exp \exp T_{n}\right)\|u(0)\|_{H^{s}} .
$$

Hence, we proved global existence in the defocusing case for (1). The focusing case is handled similarly, with the additional restriction on the mass $\|u(0)\|_{2}^{2}$, which is a conserved quantity, and is required to be small so that $\|u\|_{H^{1}}$ stays comparable to $E^{\frac{1}{2}}$ by using the Gagliardo-Nirenberg inequality. 


\section{REFERENCES}

[1] Ramona Anton. Cubic nonlinear Schrödinger equation on three dimensional balls with radial data. Comm. Partial Differential Equations, 33(10-12):1862-1889, 2008.

[2] Ramona Anton. Strichartz inequalities for Lipschitz metrics on manifolds and nonlinear Schrödinger equation on domains. Bull. Soc. Math. France, 136(1):27-65, 2008.

[3] M. D. Blair, H. F. Smith, and C. D. Sogge. Strichartz estimates and the nonlinear Schrödinger equation on manifolds with boundary. Math. Ann., 354(4):1397-1430, 2012.

[4] Matthew D. Blair, Hart F. Smith, and Christopher D. Sogge. On Strichartz estimates for Schrödinger operators in compact manifolds with boundary. Proc. Amer. Math. Soc., 136(1):247-256 (electronic), 2008.

[5] J. Bourgain. Exponential sums and nonlinear Schrödinger equations. Geom. Funct. Anal., 3(2):157-178, 1993.

[6] J. Bourgain. Fourier transform restriction phenomena for certain lattice subsets and applications to nonlinear evolution equations. I. Schrödinger equations. Geom. Funct. Anal., 3(2):107-156, 1993.

[7] J. Bourgain. Refinements of Strichartz' inequality and applications to 2D-NLS with critical nonlinearity. Internat. Math. Res. Notices, (5):253-283, 1998.

[8] N. Burq. Smoothing effect for Schrödinger boundary value problems. Duke Math. J., 123(2):403-427, 2004.

[9] N. Burq, P. Gérard, and N. Tzvetkov. On nonlinear Schrödinger equations in exterior domains. Ann. Inst. H. Poincaré Anal. Non Linéaire, 21(3):295-318, 2004.

[10] N. Burq, P. Gérard, and N. Tzvetkov. Strichartz inequalities and the nonlinear Schrödinger equation on compact manifolds. Amer. J. Math., 126(3):569-605, 2004.

[11] N. Burq, P. Gérard, and N. Tzvetkov. Bilinear eigenfunction estimates and the nonlinear Schrödinger equation on surfaces. Invent. Math., 159(1):187-223, 2005.

[12] Nicolas Burq, Patrick Gérard, and Nikolay Tzvetkov. Multilinear eigenfunction estimates and global existence for the three dimensional nonlinear Schrödinger equations. Ann. Sci. École Norm. Sup. (4), 38(2):255-301, 2005.

[13] Thierry Cazenave and Fred B. Weissler. The Cauchy problem for the critical nonlinear Schrödinger equation in $H^{s}$. Nonlinear Anal., 14(10):807-836, 1990.

[14] J. Colliander, M. Keel, G. Staffilani, H. Takaoka, and T. Tao. Global well-posedness and scattering for the energy-critical nonlinear Schrödinger equation in $\mathbb{R}^{3}$. Ann. of Math. (2), 167(3):767-865, 2008.

[15] B. Dodson. Global well-posedness and scattering for the defocusing, energy -critical, nonlinear Schrödinger equation in the exterior of a convex obstacle when $d=4$. arXiv:math/1112.0710, December 2011.

[16] J. Ginibre and G. Velo. Scattering theory in the energy space for a class of nonlinear Schrödinger equations. J. Math. Pures Appl. (9), 64(4):363-401, 1985.

[17] Martin Hadac, Sebastian Herr, and Herbert Koch. Well-posedness and scattering for the KP-II equation in a critical space. Ann. Inst. H. Poincaré Anal. Non Linéaire, 26(3):917-941, 2009.

[18] Z. Hani. A bilinear oscillatory integral estimate and bilinear refinements to Strichartz estimates on closed manifolds. arXiv:math/1008.2827, to appear in An.\&PDE, August 2010.

[19] Oana Ivanovici. Counterexamples to Strichartz estimates for the wave equation in domains. Math. Ann., 347(3):627-673, 2010.

[20] Oana Ivanovici. On the Schrödinger equation outside strictly convex obstacles. Anal. PDE, 3(3):261-293, 2010.

[21] Oana Ivanovici and Fabrice Planchon. Square function and heat flow estimates on domains, 2008. arXiv:math/0812.2733.

[22] Oana Ivanovici and Fabrice Planchon. On the energy critical Schrödinger equation in $3 D$ non-trapping domains. Ann. Inst. H. Poincaré Anal. Non Linéaire, 27(5):1153-1177, 2010.

[23] Markus Keel and Terence Tao. Endpoint Strichartz estimates. Amer. J. Math., 120(5):955-980, 1998.

[24] Herbert Koch and Daniel Tataru. Dispersive estimates for principally normal pseudodifferential operators. Comm. Pure Appl. Math., 58(2):217-284, 2005.

[25] Herbert Koch and Daniel Tataru. A priori bounds for the 1D cubic NLS in negative Sobolev spaces. Int. Math. Res. Not. IMRN, (16):Art. ID rnm053, 36, 2007.

[26] Benoit Pausader, Nikolay Tzvetkov, and Xuecheng Wang. Global regularity for the energy-critical NLS on $\mathbb{S}^{3}$, 2012. arXiv:math/1210.3842.

[27] Fabrice Planchon. Existence globale et scattering pour les solutions de masse finie de l'équation de Schrödinger cubique en dimension deux (d'après Benjamin Dodson, Rowan Killip, Terence Tao, Monica Vişan et Xiaoyi Zhang). Astérisque. Exp. No. 1042, Séminaire Bourbaki. Vol. 2010/2011.

[28] Fabrice Planchon and Luis Vega. Bilinear virial identities and applications. Ann. Scient. Éc. Norm. Sup., 42:261290, 2009.

[29] P. Smith. Conditional global regularity of Schrödinger maps: sub-threshold dispersed energy. arXiv:math/1012.4048, December 2010. 
[30] P. Smith. Global regularity of critical Schrödinger maps: subthreshold dispersed energy. arXiv:math/1112.0251, December 2011.

[31] Gigliola Staffilani and Daniel Tataru. Strichartz estimates for a Schrödinger operator with nonsmooth coefficients. Comm. Partial Differential Equations, 27(7-8):1337-1372, 2002.

[32] Robert S. Strichartz. Restrictions of Fourier transforms to quadratic surfaces and decay of solutions of wave equations. Duke Math. J., 44(3):705-714, 1977.

Laboratoire J. A. Dieudonné, UMr 7351, Université de Nice Sophia-Antipolis, Parc Valrose, F06108 Nice Cedex 02, et Institut universitaire de France

E-mail address: fabrice.planchon@unice.fr 